\title{
Low Temperature Magnetic Properties of $\operatorname{Pr}_{0.7}(\mathrm{Ca}, \mathrm{Sr})_{0.3} \mathrm{CoO}_{3}$ Oxides
}

\author{
I.G. Deac*, A. Vlădescu, I. Balasz, A. Tunyagi and R. Tetean \\ Faculatea de Fizica, Universitatea Babes-Bolyai, Str. Kogalniceanu 1,Cluj-Napoca 400084, Romania \\ We have investigated magnetic and magnetocaloric properties of $\operatorname{Pr}_{0.7}\left(\mathrm{Ca}_{1-x} \mathrm{Sr}_{x}\right)_{0.3} \mathrm{CoO}_{3}$, when the average \\ size of the interpolated cation was changed. $\mathrm{Pr}_{0.7} \mathrm{Ca}_{0.3} \mathrm{CoO}_{3}$ has an orthorhombic Pnma symmetry and it shows \\ a magnetic cluster-glass behavior below $70 \mathrm{~K}$. When $\mathrm{Sr}$ partially replaces $\mathrm{Ca}$ in this compound, its magnetic \\ properties are improved, and it begins to have ferromagnetic-like behavior. The magnetic transition temperature, \\ gradually, increases with increasing Sr content, up to $170 \mathrm{~K}$, for $x=1$. The electrical conduction also improved \\ when $\mathrm{Sr}$ content increased. All the samples show negative magnetoresitance. Magnetic entropy change $\Delta \mathrm{S}_{M}$ was \\ estimated from isothermal magnetization data. We have found that it had higher values for the samples with \\ $x>0.5$, around $1 \mathrm{~J} / \mathrm{kg} \mathrm{K}$ for $\Delta B=4 \mathrm{~T}$, with reasonable good relative cooling power.
}

PACS: 75.47.Lx, 75.30.Kz, 75.30.Sg, 73.43.Qt

\section{Introduction}

The $\mathrm{ABO}_{3}$-type transition metal oxides display remarkable magnetic and transport properties of interest for basic science as well as for technical applications (for use in solid oxide fuel cell, chemical reactors, gas separation membranes and many other applications) [1]. Doped cobaltite perovskites $\mathrm{Ln}_{1-x} \mathrm{~A}_{x} \mathrm{CoO}_{3}(\mathrm{Ln}=\mathrm{La}$, rare earth and $\mathrm{A}=\mathrm{Ca}, \mathrm{Sr}, \mathrm{Ba})$, have recently attracted much attention due to their unique feature to change the spinstate of the $\mathrm{Co}^{3+}$ (low-spin LS: $\mathrm{t}_{2 g}^{6} \mathrm{e}_{g}^{0}$, intermediate-spin IS: $\mathrm{t}_{2 g}^{5} \mathrm{e}_{g}^{1}$, high-spin HS: $\left.\mathrm{t}_{2 g}^{4} \mathrm{e}_{g}^{2}\right)$ and $\mathrm{Co}^{4+}\left(\mathrm{LS}: \mathrm{t}_{2 g}^{5} \mathrm{e}_{g}^{0}\right.$, IS: $\mathrm{t}_{2 g}^{4} \mathrm{e}{ }_{g}^{1}$, HS: $\left.\mathrm{t}_{2 g}^{3} \mathrm{e}_{g}^{2}\right)$ ions. The existence of the spin state change indicates that the difference of the electronic energies, $\delta E$, between these spin states is rather small, since the crystal field splitting of the Co-dstates and Hund's rule coupling energy are comparable for these compounds. With increasing temperature some of Co ions are progressively converted to IS or to HS state due to the small difference between Hund and crystal field energies that allows the thermal excitation of $t_{2 g}$ electrons on $\mathrm{e}_{g}$ levels [2]. This aspect was largely studied on $\mathrm{LaCoO}_{3}$ and it was found in some other cobaltites like $\mathrm{PrCoO}_{3}$ and $\mathrm{NdCoO}_{3}$, showing that the transition temperature varies as a function of the rare earth ion in the $\mathrm{LnCoO}_{3}$ series $[2,3]$. We can control the physical properties of Co oxides by controlling the value of $\delta E$, for example, by changing the ionic radius $\left\langle r_{A}\right\rangle$ of Ln, by doping A-type ions at Ln ion site. If Sr (for example) substitutes for Ln in these Co perovskites, a transition from paramagnetic phase to the ferromagnetic one takes place with increasing doping content, at about $20 \%$ [3]. $\mathrm{PrCoO}_{3}$ shows paramagnetic behavior down to $5 \mathrm{~K}$ [4]. The compound $\mathrm{Pr}_{0.7} \mathrm{Sr}_{0.3} \mathrm{CoO}_{3}$ was found to be ferro-

* corresponding author; e-mail: ideac@phys.ubbcluj.ro magnetic (below $170 \mathrm{~K}$ ) and metallic down to about 100 $\mathrm{K}$, and then semiconductor at lower temperatures [4-6]. $\mathrm{Pr}_{0.7} \mathrm{Ca}_{0.3} \mathrm{CoO}_{3}$ has a seminconductor-like (in the whole temperature range) and a magnetic cluster-glass behavior [6-9] below $70 \mathrm{~K}$. The physical properties of these compounds will depend on the average size of the A-site $\left(\mathrm{Ln}^{3+}\right.$ and $\mathrm{A}^{2+}$ ) cations, $\left\langle r_{\mathrm{A}}\right\rangle=\sum x_{i} r_{i}$ (where $x_{i}$ is the fractional occupancy of A-site ions, and $r_{i}$ is the corresponding ionic radius) $[10,11]$.

When a magnetic field is applied adiabatically on a magnetic material the temperature of the material rises, and when the field is removed the temperature decreases since the magnetic moments of the atoms become reoriented. This phenomenon is known as "magnetocaloric effect" (MCE). The latter effect is an isothermal magnetic entropy change or an adiabatic temperature change of a magnetic material upon application of a magnetic field [12]. The compounds that undergo temperature-driven paramagnetic to ferromagnetic transitions show relatively large MCE. The mixed-valency perovskites have attracted renewed interest in research due to their potential application as active magnetic materials in magnetic refrigeration techniques. Most of the studies were done on the so-called "colossal magnetoresistive manganites" [13]. Doped perovskites cobaltites have proven very useful for the study of this phenomenon, since they also have sharp paramagnetic to ferromagnetic-like transition and they are tunable by adjustment of the doping concentration. Like manganites [14], the different forms of cobaltites exhibit interesting phenomena including spin, charge and orbital ordering, electronic phase separation, insulatormetal transition, large thermoelectric power at low temperature [1-3].

In order to do a systematic analysis of how the electrical and magnetic properties can be tuned by doping, the series of cobaltites $\mathrm{Pr}_{0.7}\left(\mathrm{Ca}_{1-x} \mathrm{Sr}_{x}\right)_{0.3} \mathrm{CoO}_{3}$ was studied 
when $\left\langle r_{\mathrm{A}}\right\rangle$ gradually changes, in fine steps, from $1.305 \AA$ (for $x=0$ ) to $1.335 \AA$ (for $x=1$ ). The perovskite $A$-site is coordinated by $12 \mathrm{O}^{-2}$ ions and we used the ionic radii $R\left(\mathrm{Pr}^{3+}\right)=1.29 \AA, R\left(\mathrm{Ca}^{2+}\right)=1.34 \AA$ and $R\left(\mathrm{Sr}^{2+}\right)=1.44 \AA[14]$.

\section{Experimental details}

The $\mathrm{Pr}_{0.7}\left(\mathrm{Ca}_{1-x} \mathrm{Sr}_{x}\right)_{0.3} \mathrm{CoO}_{3}$ compounds with $x=0$, $0.2,0.5,0.7,0.8,0.95$, and 1 were prepared by conventional solid-state reaction. The mixtures of the respective oxides were calcined at $900{ }^{\circ} \mathrm{C}$ and sintered in air at $1180^{\circ} \mathrm{C}$ for 24 hours, with intermediate grindings. The powder x-ray diffraction patterns were recorded by using a Bruker D8 Advance AXS diffractometer with $\mathrm{Cu} \mathrm{K}_{\alpha}$ radiation. Data were refined by the Rietveld method using the program FULLPROF. A cryogen free VSM 12 T magnetometer (Cryogenic Ltd.) was used for magnetization and ac susceptibility measurements in the temperature range $5-300 \mathrm{~K}$ and up to $12 \mathrm{~T}$. The temperature dependent magnetization, $M(T)$, was recorded on warming in zero field-cooled (ZFC) and field-cooled (FC) modes. The resistivities were measured in another cryogen free magnet cryostat CFM-7 T (Cryogenic Ltd.) by the fourprobe technique, in the temperature range from 5 to 300 $\mathrm{K}$ and magnetic fields up to $7 \mathrm{~T}$.

The magnetocaloric effect (MCE) can be estimated by means of the magnetic entropy change $\Delta S_{M}\left(T, H_{0}\right)$. In order to do this, we measured the isothermal $M-H$ curves under different temperatures using the thermodynamic relation relation [12] (for second order phase transitions):

$$
\begin{aligned}
\Delta & S_{M}\left(T, H_{0}\right)=S_{M}\left(T, H_{0}\right)-S_{M}(T, 0) \\
& =\frac{1}{\Delta T} \int_{0}^{H_{0}}[M(T+\Delta T, H)-M(T, H)] \mathrm{d} H
\end{aligned}
$$

where $\Delta T$ is the temperature increment between measured magnetization isotherms $(\Delta T=5 \mathrm{~K}$ for our data). Generally, the important index for selecting magnetic refrigerants is based on the cooling power per unit volume, namely, the relative cooling power $(\mathrm{RCP})$ :

$$
\operatorname{RCP}(S)=-\Delta S_{M}(\max ) \delta T_{F W H M}
$$

where $\Delta S_{M}(\max )$ is the maximum magnetic entropy change and $\delta T_{F W H M}$ is the full width at half maximum of the magnetic entropy change curve [12, 13, 15].

\section{Results and discussion}

All the samples are single phase without detectable secondary phase, within the sensitivity limits of the experiment, and they have orthorhombic Pnma type structure. The x-ray diffraction pattern for a typical $x=0.5$ sample is shown in Fig. 1. Detailed results of the structural refinements are listed in Table.

When $\mathrm{Ca}^{2+}$ ions are substituted by $\mathrm{Sr}^{2+}$ ions, which have larger ionic radius, the average ionic radius of A-site $\left\langle r_{\mathrm{A}}\right\rangle$ increases with the increase in Sr concentration. As

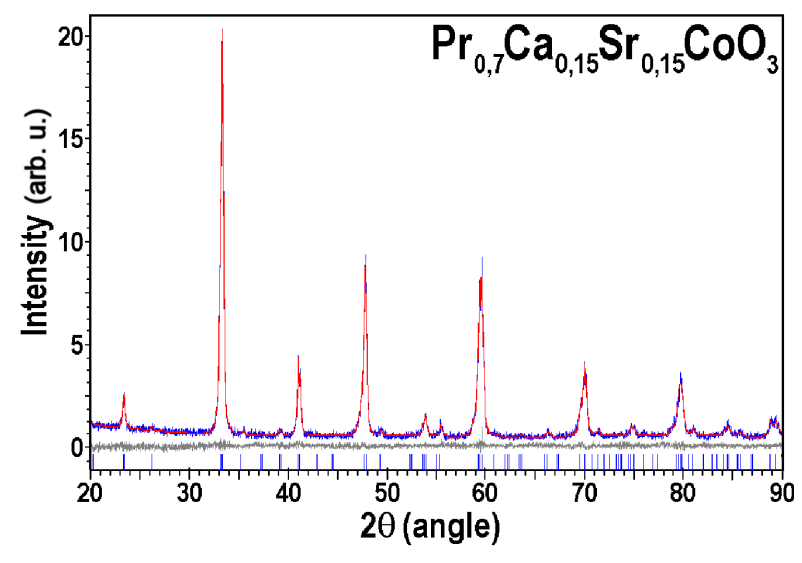

Fig. 1. Rietveld refinement plot of the x-ray diffraction data for the sample with $x=0.5$. Observed, calculated, and difference profiles are plotted on the same scale. The Bragg peaks are indicated by tick marks.

can be seen from Table, the principal effect of decreasing $\left\langle r_{\mathrm{A}}\right\rangle$ is to lower the Co-O-Co bond angle away from $180^{\circ}$, and the effect will be to reduce the overlap of the Co and $\mathrm{O}$ orbitals. The substitution of $\mathrm{Sr}$ for Ca will expand the unit cell and will make it more symmetrical.

The rapid increase of $M$ around $T_{\mathrm{C}}$ signals the phase transition from a paramagnetic to an ordered state. The temperature dependence of magnetization is strongly dependent on magnetic history starting from a temperature just below $T_{\mathrm{C}}$ to the lowest temperature with a bifurcation between ZFC and FC data at an irreversibility temperature $T_{i r r}$, as shown in Fig. 2 for the samples with $x=0,0.2,0.5$ and 0.95 . The FC branches of the temperature dependence of magnetizations exhibit typical ferromagnetic behavior for the samples with $x>0$. The ferromagnetic $T_{\mathrm{C}}$ 's of our samples, are estimated from the common inflection points of both $M_{Z F C}(T)$ and $M_{F C}(T)$ curves and they are indicated in Table. The hysteresis loops indicate that all the samples have ferromagneticlike behavior, as shown in Fig. 2 for the sample with $x=0.5$. The width of the hysteresis loop decreases markedly with increasing temperature.

The difference in the magnetization behavior between the FC and ZFC regimes reflects the energy involved in the alignment of magnetic domains in the samples suggesting that the applied field $(0.1 \mathrm{~T})$ is lower than the coercive field. The bifurcation between $M_{Z F C}(T)$ and $M_{F C}(T)$ curves is typical for doped cobaltites [3-15, 16]. No saturation was seen in $M(H)$ curves for any of the samples, even up to $12 \mathrm{~T}$. Such a behavior suggests the coexistence of a dominant ferromagnetic phase together with a non-ferromagnetic one, i.e., a phase separation scenario. We also include in this non-ferromagnetic phase, the paramagnetic contribution from the $\operatorname{Pr}^{3+}$ ions.

The ferromagnetic fraction grows with increasing $x$ for the samples with $x>0$. For the sample with $x=0$, the increase of the magnetization takes place slowly with decreasing temperature in a very large temperature range, 
suggesting a broad distribution of $T_{\mathrm{C}}$ 's. $\operatorname{Pr}_{0.7} \mathrm{Ca}_{0.3} \mathrm{CoO}_{3}$ has a behavior that is unlike of ferromagnets and is somewhat comparable to that of frustrated systems. The shape of the $M(T)$ curve for this sample was rather com- plex, not typical for pure ferromagnets, with a maximum in $M_{Z F C}(\mathrm{~T})$ at $T_{f} \sim 16 \mathrm{~K}$ and a frequency dependent maximum in ac susceptibility (not shown), both in the real part $\chi^{\prime}(T)$ and in the imaginary part $\chi^{\prime \prime}(T)[6,8]$.

TABLE

Quantitative data for the $\operatorname{Pr}_{0.7}\left(\mathrm{Ca}_{1-x} \mathrm{Sr}_{x}\right)_{0.3} \mathrm{CoO}_{3}$ compounds

\begin{tabular}{c|c|c|c|c|c|c|c}
\hline \hline$x$ & 0 & 0.2 & 0.5 & 0.7 & 0.8 & 0.95 & 1 \\
\hline$a[\AA]$ & 5.3526 & 5.3855 & 5.4024 & 5.4127 & 5.4201 & 5.4265 & 5.4268 \\
$b[\AA]$ & 7.5714 & 7.5754 & 7.5854 & 7.5915 & 7.5975 & 7.6009 & 7.6039 \\
$c[\AA]$ & 5.3526 & 5.3576 & 5.3624 & 5.3673 & 5.3704 & 5.3717 & 5.3738 \\
$V\left[\AA^{3}\right]$ & 217.427 & 218.579 & 219.751 & 220.551 & 221.156 & 221.569 & 221.901 \\
$\left\langle r_{\mathrm{A}}\right\rangle$ & 1.305 & 1.311 & 1.320 & 1.326 & 1.329 & 1.333 & 1.335 \\
$\mathrm{Co}-\mathrm{O} 1-\mathrm{Co}$ & 145.32 & 150.15 & 152.65 & 155.89 & 156.83 & 157.07 & 157.22 \\
$\mathrm{Co}-\mathrm{O} 2-\mathrm{Co}$ & 148.35 & 153.22 & 154.09 & 157.17 & 157.87 & 158.09 & 165.10 \\
$\mathrm{O} 1-\mathrm{Co}-\mathrm{O} 2$ & 119.72 & 112.23 & 110.27 & 107.99 & 107.49 & 106.99 & 84.58 \\
$T_{C}[\mathrm{~K}]$ & - & 68 & 116 & 125 & 134 & 162 & 168 \\
& & & & & & &
\end{tabular}

The ferromagnetic transition temperatures increase with increasing $\mathrm{Sr}$ content in the samples. The clusterglass behavior of the parent compound $\mathrm{Pr}_{0.7} \mathrm{Ca}_{0.3} \mathrm{CoO}_{3}$ is suppressed with increase in the substituent $\mathrm{Sr}$ ion concentrations. It seems that, the introduction of strontium in the distorted perovskite cages locally hinders the distortion of the structure. In this way, more symmetric domains will develop around the $\mathrm{Sr}^{2+}$ ion. This is the so-called "counter-distortion" effect [11], previously seen in manganites [17] It is believed that, as well as in the case of manganites, magnetic interaction of the type of double exchange between $\mathrm{Co}^{3+}$ and $\mathrm{Co}^{4+}$ ions is responsible for the ferromagnetism of cobaltites $[2,3]$. Usually, the complex spin-charge-orbital-lattice coupling results in a cluster glass behavior that is a sign of magnetic phase separation in the system, i.e. ferromagnetic order but with a possible coexistence of superparamagnetic clusters (in our case) $[5,7,16]$.

In order to evaluate the MCE, the isothermal magnetization curves of the samples were measured with a field step of $1 \mathrm{~T}$ in a range of $0-4 \mathrm{~T}$ and a temperature step of $5 \mathrm{~K}$ over a range of temperatures around $T_{\mathrm{C}}$. Such families of $M(H)$ curves are shown in Fig. 3(a) for the sample with $x=0.8$, as an example. To study the nature of the magnetic transition we built the Arrot plot and we used the Banerjee criterion [18]. In Fig. 3(b) we plot the $M^{2}$ versus $M / H$ curves (Arrot plot) where a positive slope is clearly seen in all the $M^{2}$ range, indicating that the phase transition is second order [18]. The second-order magnetic transition at $T_{\mathrm{C}}$ induces a smaller MCE but with a distribution over a broader temperature range, thus resulting in a larger $\mathrm{RCP}$.

The temperature dependences of magnetic entropy change in $1,2,3$ and $4 \mathrm{~T}$ external applied fields for the
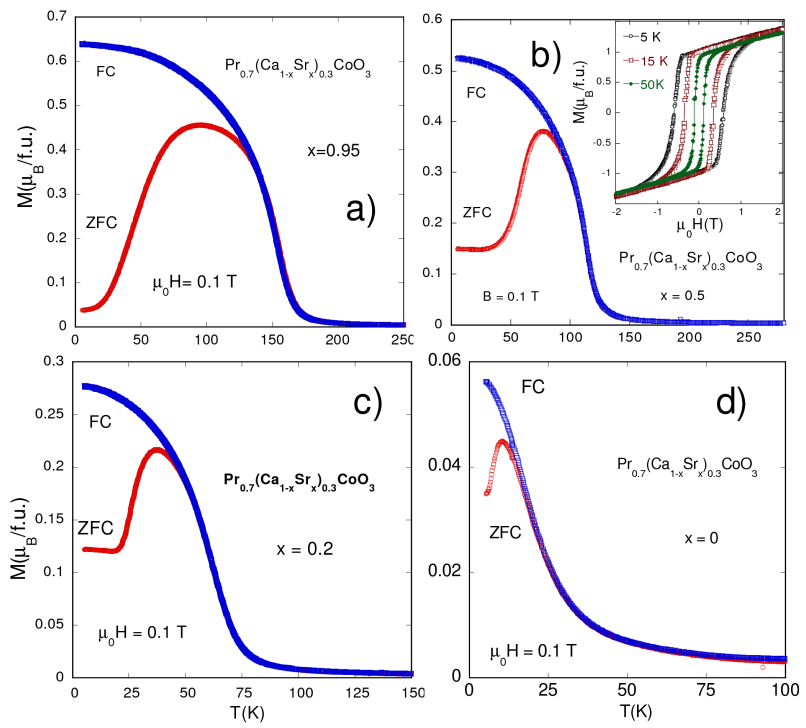

Fig. 2. Field cooled (FC) and zero field cooled (ZFC) magnetization of $\operatorname{Pr}_{0.7}\left(\mathrm{Ca}_{1-x} \mathrm{Sr}_{x}\right)_{0.3} \mathrm{CoO}_{3}$ as a function of temperature, measured in $0.1 \mathrm{~T}$. For a) $x=0.9, \mathrm{~b}$ ) $x=0.5$, (in inset: the hysteresis loops at 5, 15 and 50 $\mathrm{K})$, c) $x=0.2$ and d) $x=0$.

compound with $x=0.95$, and 0.2 are plotted in Fig. 4, as examples. The maximum values of entropy change occur almost around the transition temperatures for all the compounds. We cannot talk about a significant MCE for the sample with $x=0$ since, we have a low magnitude of magnetization and a broaden transition, with a distribution of $T_{\mathrm{C}}$ 's that lead to negligible values for $(\partial M(T, H) / \partial T)_{H}$. 

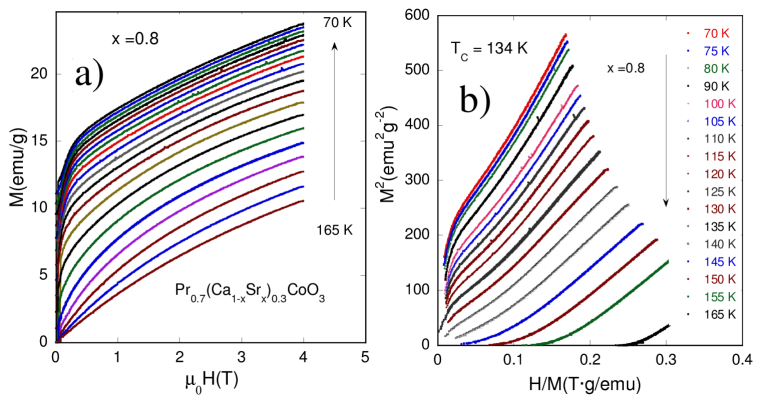

Fig. 3. (a). Isothermal magnetization curves taken at different fixed temperatures between 70 and $165 \mathrm{~K}$ for the $\mathrm{Pr}_{0.7}\left(\mathrm{Ca}_{0.2} \mathrm{Sr}_{0.8}\right)_{0.3} \mathrm{CoO}_{3}$ cobaltite. (b) Arrot plot obtained from measured $M$ vs. $H$ isotherms, for the sample with $x=0.8$.

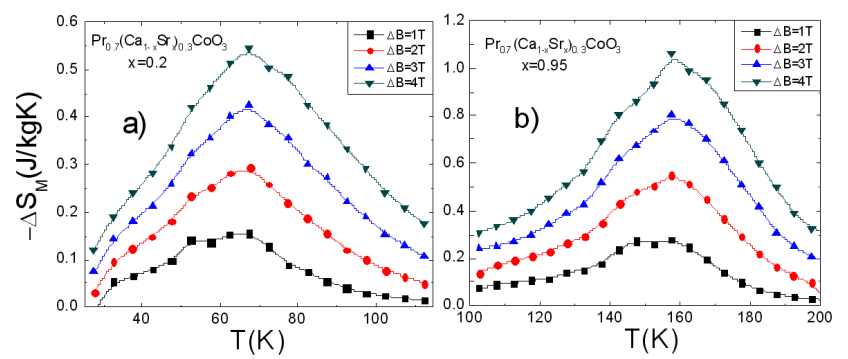

Fig. 4. The temperature dependences of the magnetic entropy change for the samples with $x=0.2$ (a) and $x=0.95(\mathrm{~b})$, in $\Delta B=1,2,3$ and $4 \mathrm{~T}$.

The maximum value of the entropy change, $-\Delta S_{M}(\max )$, is $1.065 \mathrm{~J} / \mathrm{kg} \mathrm{K}$ in $4 \mathrm{~T}$, for the sample with $x=0.95$ and it decreases linearly to $0.28 \mathrm{~J} / \mathrm{kg} \mathrm{K}$ when the field goes from 4 to $1 \mathrm{~T}$. The values of the maximum of the entropy change, $-\Delta S_{M}(\max )$, are almost the same for $x>0.5$, while the widths at the half maximum gradually increase from $51 \mathrm{~K}$, for $x=1$ to $63 \mathrm{~K}$ for $x=0.5$ in $4 \mathrm{~T}$. The relative cooling power, RCP is maximum for the sample with $x=0.8$ being $54.9 \mathrm{~J} / \mathrm{kg}$ for $\Delta B=4 \mathrm{~T}, 44.8 \mathrm{~J} / \mathrm{kg}$ for $\Delta B=3 \mathrm{~T}$, $30.2 \mathrm{~J} / \mathrm{kg}$ for $\Delta B=2 \mathrm{~T}$ and $15.7 \mathrm{~J} / \mathrm{kg}$ for $\Delta B=1 \mathrm{~T}$. The sample with $x=0.5$ has RCP's as $53.5 \mathrm{~J} / \mathrm{kg}$, $41.6 \mathrm{~J} / \mathrm{kg}, 29.4 \mathrm{~J} / \mathrm{kg}$ and $15.2 \mathrm{~J} / \mathrm{kg}$, respectively. By using these $(\mathrm{Ca}, \mathrm{Sr})$ dopings, with $x>0.2$, the relative cooling powers will not change very much, but they could be used in different temperature ranges. These values for RCP are somewhat smaller than those obtained in doped manganite perovskites but they are high enough for technical interest.

The samples have semiconducting behavior at low temperatures, in spite of their very low values of the electrical resistivity. In Fig. 5 is described the temperature dependence of resistivity in zero applied magnetic field for the samples with $x=0.2,0.5,0.8$ and 0.95 (for clarity). The samples with $x>0.5$ have metallic behavior down to about $100 \mathrm{~K}$, and then they have semiconductor behavior $(\mathrm{d} \rho / \mathrm{d} T<0)$ at lower temperatures, probably, due to a grain boundary effect [19]. The samples with higher $\mathrm{Ca}$ content have semiconductor behavior in the whole temperature range. It can be seen that the value of the resistivity decreases with increasing Sr content in the samples. This behavior suggests, again, that more symmetric domains will develop around the $\mathrm{Sr}^{2+}$ ions allowing a better overlapping of the Co and $\mathrm{O}$ orbitals. There is no signature of the magnetic phase transition on the temperature dependence of resistivity. This decoupling of magnetization and transport is unusual in perovskites, where the double exchange mechanism presumes a link between electron hopping and magnetic moments alignment $[1,14]$. A possible explanation for this can reside in the electron scattering mechanism at the grain boundaries, which can mask the change in the electrical conduction due to the magnetic phase transition [19].

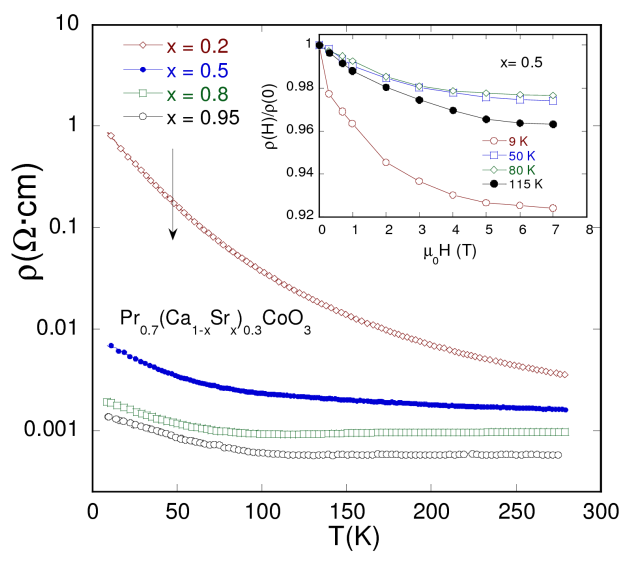

Fig. 5. The temperature dependence of electrical resistivity in zero applied magnetic field, for the samples with $x=0.2,0.5,0.8$ and 0.95 . In inset: $\rho(H) / \rho(0)$ measured at $9 \mathrm{~K}, 50 \mathrm{~K}, 80 \mathrm{~K}$ and $150 \mathrm{~K}$ for the sample with $x=0.95$.

All the samples showed negative magnetoresistence. For the samples with high Ca content the magnetoresitence is maximum at the lowest temperature and in 7 $\mathrm{T}$, as can be seen in the inset of Fig. 5. In this case, the magnetoresitance $M R=1-\rho(H) / \rho(0)$ can be about $7.6 \%$, at $9 \mathrm{~K}$ and $7 \mathrm{~T}$, for the sample with $x=0.5$. For the other samples, $M R$ at $9 \mathrm{~K}$ and $7 \mathrm{~T}$ is not higher than $3 \%$. Thus, the larger $M R$ observed for these samples at $9 \mathrm{~K}$ can be interpreted as tunnelling magnetoresistance $(T M R)$ effect due to the increase of the intergrain insulating barriers, as it was found in some other transition metal oxides [20]. A better coupling between transport and magnetic properties can be found in the sample with $x=0.95$, where the maximum $M R$ is about $5 \%$ in $7 \mathrm{~T}$ and $150 \mathrm{~K}$, while for the sample with $x=1$ it was about $3.4 \%$ close to the magnetic transition.

The decrease of resistivity and the enhancement of the magnetic properties with increasing Sr content in the samples suggest that these properties can be correlated, as in the case of the manganite perovkites where 
the double exchange interaction improves the electrical conduction. The magnetotransport data can be described in terms of percolative transport through some ferromagnetic-like, metallic regions, in the presence of grain boundary scatterings $[19,20]$.

\section{Conclusions}

High-quality samples of $\operatorname{Pr}_{0.7}\left(\mathrm{Ca}_{1-x} \mathrm{Sr}_{x}\right)_{0.3} \mathrm{Co}_{x} \mathrm{O}_{3}$ $(x=0,0.2,0.5,0.8,0.95$, and 1$)$ were prepared by standard ceramic reaction, and their magnetic and electrical properties were investigated. All the compounds crystallize in an orthorhombic structure. The samples with $x>0$ show ferromagnetic-like behavior (ferromagnetic order but with a possible coexistence of superparamagnetic clusters) below a transition temperature, that decreases with decreasing Sr content in the samples. $\mathrm{Pr}_{0.7} \mathrm{Ca}_{0.3} \mathrm{CoO}_{3}$ has cluster-glass behavior, with no long range magnetic order. When $\mathrm{Sr}$ partially replaces $\mathrm{Ca}$ in this system, the cluster-glass behavior is suppressed and the magnetism is enhanced. Similarly, the electrical conduction is improved with increasing Sr content in the samples. All the samples have negative magnetoresistance at low temperatures. The electrical conduction is percolative and controlled by grain boundary effects. The maximum magnetic entropy change, $-\Delta S_{M}(\max )$, for the samples with $x>0.5$, is around $1 \mathrm{~J} / \mathrm{kg} \mathrm{K}$, while $\mathrm{RCP}$ is around $54 \mathrm{~J} / \mathrm{kg}$, for $\Delta B=4 \mathrm{~T}$. By (Ca,Sr) doping in this system we can obtain rather high cooling powers in various temperature ranges, with reasonably good magnetocaloric values.

\section{Acknowledgments}

This work was supported by the grant PNII-IDEI code 2590/2008 of CNCSIS-UEFISCSU, Romania.

\section{References}

[1] J.B. Goodenough, Rep. Prog. Phys. 67, 1915 (2004).

[2] M.A. Señarís-Rodríguez, J.B. Goodenough, J. Solid State Chem. 118, 323 (1995).

[3] J.-Q. Yan, J.-S. Zhou, J.B. Goodenough, Phys. Rev. B 69, 134409 (2004).

[4] H.W. Brinks, H. Fjellvag, A. Kjekshus, B.C. Hauback, J. Solid State Chem. 147, 464 (1999).

[5] C. Leighton, D.D. Stauffer, Q. Huang, Y. Ren, S. El-Khatib, M.A. Torija, J. Wu, J.W. Lynn, L. Wang, N.A. Frey, H. Srikanth, J.E. Davies, Kai Liu, J.F. Mitchell, Phys. Rev. B 79, 214420 (2009).

[6] I.G. Deac, A. Vlădescu, I. Balasz, A. Tunyagi, R. Tetean, Int. J. Mod. Phys. B 24, 762 (2010).

[7] A.K. Kundu, P. Nordblad, C.N.R. Rao, J. Solid State Chem. 179, 923 (2006).

[8] I.G. Deac, R. Tetean, D. Andreica, E. Burzo, IEEE Trans. Magn. 44, 2922 (2008).

[9] T. Fujita, T. Miyashita, Y. Yasui, Y. Kobayashi, M. Sato, E. Nishibori, M. Sakata, T. Adachi, Y. Ohishi, M. Takata J. Phys. Soc. Jpn. 73, 1987 (2004).

[10] J.P. Attfield, Cryst. Eng. 5, 427 (2002).

[11] B. Raveau, Phil. Trans. R. Soc. A 366, 83 (2008).

[12] A.M. Tishin, Y.I. Spichkin, The Magnetocaloric Effect and Its Applications, Institute of Physics, Bristol 2003.

[13] M.H. Phan, S.C. Yu, J. Magn. Magn. Mater. 308, 325 (2007).

[14] Y. Tokura, Rep. Prog. Phys. 69, 797 (2006).

[15] V.K. Sharma, M.K. Chattopadhyay, S.B. Roy, J. Phys. D: Appl. Phys. 40, 1869 (2007).

[16] H.M. Aarbogh, J. Wu, L. Wang, H. Zheng, J.F. Mitchell, C. Leighton, Phys. Rev. B 74, 134408 (2006).

[17] D. Zhu, P. Cao, W. Liu, X. Ma, A. Maignan, B. Raveau, Materials Letters 61, 617 (2007).

[18] S.K. Banerjee, Phys. Lett. 12, 16 (1964).

[19] M. Ziese, Rep. Prog. Phys. 65,143 (2002).

[20] A.K. Kundu, B. Raveau, arXiv:1005.5426v1 [condmat.mtrl-sci] (2010). 